\title{
PERCEPÇÃO DOS CUIDADORES FRENTE ÀS DIFICULDADES ENCONTRADAS NO CUIDADO DIÁRIO DE IDOSOS DEPENDENTES INSTITUCIONALIZADOS
}

\author{
Pâmela Billig Mello \\ Aline Martinelli Piccinini ${ }^{2}$ \\ Patrícia Viana da Rosa ${ }^{3}$ \\ Luís Henrique Telles da Rosa 4 \\ Solange B. Billig Garcês 5
}

\section{Resumo}

A saúde do idoso depende muito de cuidados, pois a velhice torna as pessoas mais vulneráveis e sujeitas à necessidade de auxílio. $\mathrm{O}$ objetivo deste estudo foi verificar a percepção dos cuidadores frente às dificuldades encontradas no cuidado diário de idosos dependentes institucionalizados. O estudo foi realizado na cidade de Cruz Alta (RS), no Asilo Santo Antônio, uma instituição filantrópica dirigida pela Conferência dos Vicentinos e administrada internamente por religiosas. A amostra foi constituída por sete cuidadores de idosos da instituição. Os instrumentos utilizados foram um formulário de identificação e uma entrevista semi-estruturada com o cuidador, buscando verificar o que ele pensa sobre o envelhecimento, bem como a forma como atua frente às dificuldades de cuidado do idoso dependente institucionalizado. As informações foram gravadas e posteriormente transcritas. Para a análise das informações qualitativas, utilizou-se a técnica da análise de conteúdo. As respostas indicam que os cuidadores têm uma visão do seu cuidado com os idosos como sendo algo definido pela experi-

\footnotetext{
1 Fisioterapeuta, mestre em Fisiologia pela Universidade Federal do Rio Grande do Sul (UFRGS), doutoranda em Fisiologia pela UFRGS. E-mail: panmello@hotmail.com

2 Fisioterapeuta, mestranda em Docência pela Universidade Nacional Tecnológica (UNT) da Argentina e em Ciências do Movimento Humano pela UFRGS. E-mail: aaline-martinelli@hotmail.com

3 Professora da Universidade de Cruz Alta (Unicruz), doutora em Gerontologia Biomédica pela Pontifícia Universidade Católica do Rio Grande do Sul (PUC/RS). E-mail: patriciarosa1@hotmail.com

4 Professor da Universidade Federal de Ciências da Saúde de Porto Alegre (UFCSPA), doutor em Gerontologia Biomédica pela PUC/RS. E-mail: 1htr@comnet.com.br

5 Professora da Unicruz, mestre em Ciências do Movimento Humano pela Universidade Estadual de Santa Catarina (Udesc), doutoranda em Ciências Sociais pela Universidade do Vale dos Sinos (Unisinos). E-mail: sbgarces@hotmail.com

Grupo Interdisciplinar de Estudos do Envelhecimento Humano.

Fomento: Programa Interinstitucional de Bolsas de Iniciação Científica - Pibic/Unicruz 2004-2005.
} 
ência, e não como um processo de trabalho e aperfeiçoamento. Com relação à concepção de envelhecimento referida pelos cuidadores, percebe-se que está relacionada com os aspectos negativos da velhice, descrevendo o idoso como um ser dependente, principalmente devido ao seu vínculo com a institucionalização. Os relatos apontam, ainda, uma imagem de infantilização do envelhecimento. Ao final, o estudo indica que as concepções dos cuidadores entrevistados sobre o processo do envelhecimento são identificadas principalmente pela dependência e o cuidado definido pela experiência de vida de cada cuidador.

Palavras-chave: Envelhecimento. Concepção. Experiência. Institucionalização. Dependência.

\section{Considerações Iniciais}

Uma das características deste século é o aumento da expectativa de vida, que cresceu muito entre os anos 1960 e 1990 em muitos países (WALSH, 2004). Segundo Grimley Evans (1997), isto pode ser considerado resultado das melhores condições de vida e da diminuição da mortalidade infantil.

O aumento da expectativa de vida é uma conquista de muitas nações, principalmente entre aquelas que se encontram em processo de desenvolvimento, como o Brasil. Por volta do ano de 2025, o Brasil terá cerca de 34 milhões de indivíduos com sessenta anos ou mais. Esta perspectiva, nos últimos anos, promoveu um aumento no interesse por pesquisas relacionadas aos efeitos proporcionados pelo envelhecimento. Já observamos algumas novas tendências; porém, antes de tudo é preciso notar que não está ocorrendo apenas o aumento da população idosa, mas um aumento da duração e permanência na idade da velhice. Entretanto, este feito não se reveste apenas de vantagens ou benignidade, visto que sua conseqüência revela fatores negativos, como os associados à falta de planejamento político-social para abarcar este contingente populacional (CRUZ; ALHO, 2000).

O envelhecimento populacional é uma tendência que traz novas demandas sociais, políticas e econômicas (WORLD HEALTH ORGANIZATION, 2002). O aumento do número e da proporção de idosos traz muitas conseqüências na área social, e não menos na da saúde (KHAW, 1997).

Neri (2001) salienta que a relação entre idade cronológica e envelhecimento é apenas um elemento indicador neste processo. Desta forma o critério cronológico é uma referência frente a um mundo temporalizado. Os eventos marcadores dos vários períodos do ciclo vital dependem de parâmetros sociais combinados com critérios de outras disciplinas como 
Psicologia, Biologia e das Ciências Sociais. O envelhecer acontece associado ao processo de desenvolvimento, sendo influenciado por uma complexa combinação de fatores ao longo de toda a vida.

Questionamo-nos, então, qual seria a melhor definição de envelhecimento? O conceito varia para uma visão biologicista ou social (STREY et al., 1999), mas o fato é que o envelhecimento delimita mudanças biológicas e sociais (RODRÍGUEZ, 1994) que vão desde as alterações fisiológicas do organismo - como nos sistemas muscular, ósseo, nervoso, circulatório, pulmonar, endócrino, imunológico e epitelial, embora estas alterações variem entre os diversos tecidos e entre diferentes indivíduos (FARO JÚNIOR; LOURENÇO; BARROS NETO, 1996) - até as alterações sociais e culturais (FERNANDEZ-BALLESTEROS, 1994), que definem diferentes direitos e responsabilidades e o papel social do idoso na sociedade (RODRÍGUEZ, 1994).

Diante desse quadro de aumento da população idosa combinado com as necessidades desta parcela da população e as características de vida de agrupamentos familiares em centros urbanos, percebe-se a necessidade da existência de instituições de diversos tipos (lar/casa/asilo para idosos, hospital de apoio, centro-dia) que pudessem atender/hospedar, por períodos limitados ou não, tais idosos, em decorrência da dificuldade/incapacidade dos familiares/cuidadores. Em todo o mundo, inclusive no nosso meio, o número destes tipos de instituições tem aumentado (CHAIMOWICZ; GRECO, 1999).

O que se percebe é que as instituições para idosos são desvalorizadas. Por exemplo, a palavra asilo lembra pobreza e rejeição, contém uma carga negativa, sendo geralmente empregada quando nos referimos a uma instituição destinada a idosos carentes (REZENDE, 2002).

Embora a maioria da população idosa seja independente e resida na comunidade, uma minoria precisa contar com o apoio de instituições residenciais de longa permanência. Essa minoria, na maioria das vezes, é constituída de idosos muito desvalidos, que acumulam problemas sociais e médicos.

A decisão pelo asilamento configura um drama social. Mais do que isso, a vinda para o asilo, reflete Graeff (2007), leva-nos a ver a velhice enquanto problema social e a pensar sobre os riscos próprios do envelhecimento, especialmente as doenças mais comuns dessa idade.

Mas vir para o asilo também pode ser um projeto do idoso. Ao invés de permanecer em casa, ocupando um espaço do qual ele não se sente parte, 
o idoso renuncia à casa e opta por recuperar, parcial ou totalmente, a sua autonomia no espaço asilar (GRAEFF, 2007).

Em 2002, o número de idosos residentes em instituições de longa permanência era de 19 mil em todo o país, segundo o Relatório da V Caravana Nacional de Direitos Humanos (CARAVANA . . . 2002). Já os dados da Gerência de Atenção à Pessoa Idosa do Ministério de Desenvolvimento Social relatam que, em 2004, já se somavam 24.964 idosos utilizando as Instituições de Longa Permanência vinculadas ao Ministério (CAMARANO, 2004).

É sabido que a instituição é o último recurso que se busca para o atendimento sócio-sanitário ao idoso. Sabe-se também que a questão da assistência para idosos em instituições como estas, são de altos custos e grandes dificuldades de manutenção. No Brasil, a maioria das instituições é filantrópica e conta com o apoio de congregações religiosas da comunidade. Dessa forma, as instituições encontram poucos recursos, principalmente em relação a pessoal qualificado/capacitado para o cuidado de idosos (VAGETTI; WEINHEIMER; OLIVEIRA, 2007).

A preservação da qualidade de vida frente às ameaças de restrição da autonomia e da independência causadas pelo empobrecimento da vida social e pela deterioração da saúde são desafios que a longevidade propõe aos idosos (DUARTE; DIOGO, 2000).

Os estudos na comunidade e em instituições permitem os conhecimentos demográficos e epidemiológicos, fornecendo dados importantes para pesquisas sobre este processo (SINÉSIO; ANDRADE, 2000).

A probabilidade de uma pessoa necessitar de internação em uma instituição, após os 65 anos, é estimado em 43\%, sendo que somente em torno de $5 \%$ são de fato admitidos na instituição. A utilização de uma casa de repouso cresce para $6 \%$ na faixa entre 75 e 84 anos, e para $22 \%$ na faixa acima de 85 anos.

Katz (1996) estudou 1,3 milhões de idosos que viviam em casa de repouso e observou que, dentre eles, 16\% tinham entre 65 e 74 anos; $39 \%$ entre 75 e 84 anos; e $45 \%$ tinham 85 anos ou mais. Dentro deste grupo, $40,3 \%$ a $91 \%$ apresentavam algum tipo de dificuldade em atividades de vida diária.

A maioria dos idosos mora em sua própria casa ou na casa de um dos filhos. Este tipo de arranjo funciona razoavelmente, enquanto o idoso é capaz de cuidar de si mesmo. Porém, quando ele passa a necessitar de ajuda para o cuidado podem surgir muitas dificuldades, que sobrecarregam especialmente o cuidador familiar principal. Entretanto, devido às mudan- 
ças acima citadas, nem sempre a família tem condições de cuidar do idoso e necessita interná-lo em asilos, em caráter temporário ou permanente (QUEIROZ, 2000).

Um estudo sobre o suporte domiciliar aos adultos com perda de independência (CALDAS, 2003) aponta que mais de $90 \%$ das famílias não receberam ajuda de serviços, organizações ou grupos voluntários e/ou agências particulares. Deste modo, constata-se que os cuidados oriundos de redes informais de apoio constituem a mais importante fonte de suporte a idosos, merecendo destaque a figura do cuidador (MONTEIRO; FARO, 2006).

Os cuidadores são pessoas que cuidam de pessoas mental ou fisicamente incapacitadas. Ao falar sobre o cuidar de idosos, Creutzberg (2000) classifica o cuidado em quatro níveis: o autocuidado, o cuidado informal, o cuidado formal e o cuidado profissional.

$\mathrm{O}$ autocuidado envolve um conjunto de ações desenvolvidas pelo indivíduo em seu benefício. O cuidado informal constitui em uma ação exercida por familiares, vizinhos ou amigos sendo a forma mais comumente encontrada junto à assistência a idosos no Brasil. Os cuidadores informais são formados pelos familiares e amigos. Quase sempre estes não possuem nenhuma capacitação, ajuda ou apoio adicional. Também não possuem auxílio financeiro ou reconhecimento. Em algumas situações, podem inclusive não ter tido uma relação prévia boa com o paciente. Muitas vezes, estes apresentam, ou passam a apresentar seus próprios problemas de saúde, não reconhecendo o efeito deste cuidado sobre sua saúde física e mental.

O cuidado formal envolve ações prestadas por uma rede de serviços de saúde pública e privada ao atendimento do idoso, e a institucionalização que se torna necessária quando os cuidados exigem uma maior complexidade. Os cuidadores profissionais incluem o cuidado de enfermaria, o cuidado a domicílio, serviços voluntários, clubes e centros de cuidado diurno. Este tipo de cuidador pode trabalhar com um grande grupo de pessoas com uma variada capacidade e recursos.

Cada tipo de cuidador (familiar, social, privado, governamental) funciona sob diferentes limites e motivações. Para Boff (1999) o cuidado aparece quando existe uma preocupação com algo ou alguém, quando sua existência é importante, participamos de sua vida e, portanto, cuidamos. De imediato observamos que cuidar envolve relações objetivas e subjetivas que escapam de lógicas racionais e mensuráveis.

$\mathrm{O}$ cuidado institucional pode ser necessário devido à falta de um cuidador, falta de controle esfincteriano, habitação inapropriada, serviços locais pobres, incapacidade de tal nível que requer atenção médica ou de 
enfermagem diária, desejo do paciente, necessidade da família trabalhar ou custos elevados do cuidado, podendo o Estado oferecer incentivos financeiros (SILVA, 2000a; GUNTHER, 2000).

O cuidado é uma das essências que integra e estrutura o ser humano. Como tal, é imprescindível na promoção da vida, da saúde e manutenção da espécie. No caso da população idosa, o cuidado às necessidades de saúde requer o desenvolvimento de um corpo de conhecimento multidisciplinar através de práticas de assistência e de pesquisa em diversos contextos de vida (VAGETTI; WEINHEIMER; OLIVEIRA, 2007).

As instituições de longa permanência devem cumprir essa dupla função de ser um lugar para os idosos viverem e de proporcionar os cuidados de que necessitam (KANE; KANE, 1987).

Silva (2000b) critica a incapacidade, a falta de habilidade dos profissionais da saúde em cuidar de sujeitos com doenças graves ou terminais. Esta incapacidade e a proximidade da morte, segundo o autor, nos amedrontam.

A maioria das instituições para idosos é filantrópica, têm visão caritativa e são geralmente mantidas por associações religiosas (espíritas, católicas, evangélicas), por associações ou organizações beneficentes. Assim, muitas vezes, essas instituições não têm infra-estrutura adequada nem profissionais qualificados para atender os idosos, existindo também instituições clandestinas que constituem verdadeiros depósitos de velhos (MENDONÇA, 2006).

Born e Boechat (2002) ressaltam que a qualidade do cuidado aos idosos no ambiente asilar pode ser determinante na vontade de viver e na saúde dessas pessoas. Alertam, porém, para a realidade na maioria das instituições, que são desqualificadas, com estrutura constrangedora que despersonaliza o idoso e afasta-o do convívio familiar e social.

A satisfação em cuidar de pessoas idosas é determinada por muitos fatores sociais, físicos e financeiros, entre outros (BORG; HALLBERG; BLOMQVIST, 2006). O sentimento de solidão, o decréscimo da capacidade de autocuidado, a saúde mais frágil, a ansiedade e os poucos recursos financeiros dos idosos são fatores que devem ser considerados no cuidado diário a esta parcela específica da população com a intenção de preservar ou melhorar sua qualidade de vida (PRATO et al., 2007).

Segundo Caldas (2003), existem poucos estudos no Brasil sobre as demandas do cuidador do idoso, já que este personagem ainda é um desconhecido no cenário público. Diante desse quadro atual, o objetivo deste estudo foi verificar a percepção dos cuidadores frente às dificuldades en- 
contradas no cuidado diário de idosos dependentes institucionalizados, bem como verificar a percepção acerca do envelhecimento que esses profissionais apresentam.

\section{Caminho Metodológico}

As informações sócio-demográficas que caracterizam a amostra são apresentadas em números absolutos. A investigação utilizou uma abordagem qualitativa para analisar aspectos da atuação do cuidador diante das dificuldades encontradas no cuidado de idosos dependentes em uma unidade de atendimento asilar.

O estudo foi desenvolvido no município de Cruz Alta, estado do Rio Grande do Sul, situado na região noroeste do estado, tendo como instituição de cuidado ao idoso o Asilo Santo Antônio. O Asilo foi fundado em 1940 com o objetivo de abrigar pessoas idosas carentes, inválidas e/ou desamparadas.

O Asilo Santo Antônio é uma Instituição filantrópica, dirigida pela Conferência dos Vicentinos e administrada internamente por religiosas da Ordem das Irmãs Franciscanas Missionárias de Maria Auxiliadora, sendo mantida através de doações da comunidade. Possui capacidade de abrigar em torno de 80 idosos, mas na época da pesquisa abrigava de fato 60 idosos. Está localizado junto ao centro do município de Cruz Alta, sendo a única instituição para abrigo aos idosos do município.

Como o objetivo deste trabalho é conhecer a percepção dos cuidadores em relação ao envelhecimento e às dificuldades encontradas no cuidado diário de idosos dependentes institucionalizados, a população deste estudo foi constituída pelos cuidadores dos idosos institucionalizados no Asilo. A amostragem foi por conveniência, objetivando a participação de todos os cuidadores da instituição-alvo que preenchessem os critérios de inclusão e aceitassem participar.

Foram critérios de inclusão ser responsável pelo cuidado direto dos idosos e ter tido contato com os idosos em pelo menos 20 dos últimos 30 dias.

Os instrumentos de coleta dos dados foram um questionário fechado para identificação do cuidador e uma entrevista em profundidade semiestruturada contendo questões abertas norteadoras, com o objetivo de conhecer o que o cuidador pensa, como concepções sobre o idoso e sobre o envelhecimento, bem como a forma como atua frente às dificuldades no cuidado do idoso dependente institucionalizado. 
Inicialmente, os cuidadores foram informados sobre os objetivos do estudo e que, em nenhum momento, nomes ou fotos das pessoas seriam utilizados ou divulgados. Os cuidadores que aceitaram participar do estudo assinaram um termo de consentimento livre e esclarecido, no qual constavam os objetivos do trabalho, implicações quanto a sua participação ou não, informação quanto à desistência do mesmo e o modo de contato com os pesquisadores. $\mathrm{Na}$ assinatura, os participantes manifestaram a disposição de participar do estudo e permitiram a utilização das informações colhidas no estudo.

Para análise das informações qualitativas, foi utilizada a técnica de análise de conteúdo de Bardin (1979) que compreendeu uma pré-análise, incluindo leitura global das entrevistas para ter um primeiro contato com o conteúdo que elas veiculavam, exploração do material, identificando as unidades de registro em cada entrevista, e procurando interpretar com base na discussão teórica.

\section{Resultados}

Nos resultados são apresentadas, primeiramente, as características da população em estudo e posteriormente são analisadas as questões relativas ao objeto de estudo deste artigo: a percepção dos cuidadores sobre o envelhecimento e as dificuldades no cuidado de idosos institucionalizados.

\subsection{Características Sócio-Demográficas da População}

Para análise das informações sobre as características sócio-demográficas utilizou-se um questionário como forma de identificar os cuidadores dos idosos dependentes institucionalizados do asilo Santo Antônio da cidade de Cruz Alta. Todos os cuidadores entrevistados eram do sexo feminino, com uma média de idade de 50,71 anos.

Foram entrevistadas sete cuidadoras. Destas, três eram freiras, três casadas e uma separada. Em relação ao nível de escolaridade, uma tinha o ensino fundamental incompleto, quatro tinham o ensino médio completo e duas o ensino médio incompleto.

As cuidadoras dedicavam-se somente ao trabalho com os cuidado destes idosos, com exceção de uma, que tinha também um cargo de direção na mesma instituição. Além disso, todas as cuidadoras possuíam um bom tempo de experiência no cuidado com idosos institucionalizados, apresentando uma média de 12 anos de prestação deste tipo de serviço. 


\subsection{Percepções dos Cuidadores}

Na segunda parte do trabalho, para a análise das informações qualitativas, utilizou-se a técnica da análise de conteúdo de Bardin (1979), conforme descrito anteriormente.

\subsubsection{A Qualidade do Cuidado como Algo Definido pela Experiência}

As respostas indicaram que a maioria dos cuidadores tem a idéia de que seu cuidado depende da experiência que eles têm com os idosos, ignorando a necessidade de qualificação para tal função.

[ ... ] a gente tira uma lição de vida $[\ldots]$ tudo o que a gente fizer na vida da gente mais tarde vai se refletir de uma maneira ou de outra $[\ldots]$.

É isso aí, o dia-a-dia, uma rotina [de cuidado], não tem o que melhorar mais.

Para alguns, a melhora da qualidade do cuidado que oferecem aos idosos depende muito mais de fatores subjetivos, como atenção, carinho ou tempo dispensado aos idosos, do que de qualificação adequada para tal função.

Eu acho assim que, se a gente tivesse mais, assim, tempo pra ficar com eles, assim, para ficar ali, conversando, dando a atenção merecida pra eles, eu acho que seria melhor, é bem puxado, eu acho que seria interessante.

Por outro lado, poucos cuidadores, os com maior grau de escolaridade, demonstraram em suas falas a necessidade de uma preparação adequada para o cuidado de idosos, enfatizando sempre a importância de estar preparado para lidar com as doenças da velhice.

Faz muitos anos que a gente não fez mais cursos, os casos mais graves que eu tenho aqui é Alzheimer, e acabamos só lendo algumas reportagens do jornal da cidade sobre isso.

Hoje em dia, tem muitos cursos com geriatrias, é um ramo importante para uma palestra, ou um curso, na área do idoso, porque por mais que tu adquira experiência em cuidar, 
as coisas novas ajudam, a troca de experiências, porque é importante a gente se refazer todo dia.

3.2.2 A Velhice como uma Etapa de Dependência: o velho como uma criança

Com relação à concepção do envelhecimento, percebe-se na fala dos cuidadores, que o envelhecimento está relacionado com a velhice, com o ser velho, e não é visto como um processo natural que todos estamos sujeitos a vivenciar em um determinado período de nossas vidas.

Pode-se perceber que todos os cuidadores descrevem o idoso como um ser dependente, devido ao seu vínculo com a institucionalização, comparando o idoso com uma criança que necessita de cuidado e atenção.

[... ] ele se torna uma pessoa frágil, uma pessoa limitada, bem mais sensível e carente, ele volta a ser novamente uma criança $[\ldots]$.

Eu acho que a pessoa, assim, idosa, ela fica mais dependente, ela depende mais das pessoas, e aí a pessoa idosa tem que aprender a ser dependente de novo porque uma vez na vida ela já foi dependente quando criança e depois eles voltam a ser dependente. É uma coisa difícil pra eles voltar a ser dependente e às vezes eles têm uma certa dificuldade. Eles sabem que vão depender de uma outra pessoa pra ajudar eles a tomar banho, ajudar a vestir uma roupa, assim, depois de muito tempo, às vezes precisa até que tu dê comida na boca pra eles $[\ldots]$.

Pra mim é bom [trabalhar com idosos] porque a gente tá direto aprendendo as coisas com eles; é também como tu trabalhar com um criança [ . . . ]. É como eu tô te dizendo, o idoso é que nem uma criança porque eles regridem bem pra trás. Isso é negativo, porque eles regridem bem mais pra trás porque criança vai pra frente e o idoso vai pra trás. 


\subsubsection{O Envelhecer como Algo Distante}

Verificou-se, também, que os cuidadores não relatam pensar sobre a sua velhice. Convivem com idosos, mas não se colocam nesta posição no futuro.

[... ] que eu fique bem velhinha assim e que eu aceite $[\ldots]$ mas eu nunca parei pra pensar quando eu ficar velha $[\ldots]$.

Ah, eu pretendo não ficar como um idoso desses [refere-se aos idosos dos quais cuida], porque a gente tem que ajudar eles na limpeza, porque eles regridem bem mais. A gente aprende aqui muitas coisas; a gente tem que se ajudar, também, pra não chegar no ponto deles.

Depois que estou lá nos 70, 80 que eu vou começar me preparar, é agora que eu tenho que acolher as limitações [...]. Aceitando as limitações, justamente porque eu tenho problema de reumatismo, nas articulações, então, não adianta eu achar que o médico vai fazer milagres no sentido assim que é pra mim não abraçar tanto o trabalho [ . . . ]. Às vezes eu ficou aqui pensando, eu não sei o que vai ser o dia de amanhã, se eu vou ficar velha, se eu vou morrer de repente e aí eu vou planejar, é só perder tempo... Eu acho que a minha geração não vai até tão longe...

Os relatos acima refletem uma realidade atual. Não pensamos e não valorizamos a velhice. D'Alencar (1998) explicita que é necessário preparar gerações, independentemente da idade, para valorizar as experiências, a memória, as emoções das pessoas e a sabedoria de vida adquirida ao longo do tempo.

\section{Considerações Finais}

Atualmente, o entendimento acerca do que seja velhice vem se modificando e, mesmo que lentamente, vem estabelecendo uma nova relação da nossa cultura com o envelhecimento (LEITE, 2005). Beauvoir (1990) explicita que o processo de envelhecimento vai acontecendo progressivamente com o passar dos anos, sem que as pessoas jovens percebam as modificações 
que ocorrem ao longo do tempo, e menciona que é o olhar do outro que vê o quanto um determinado indivíduo envelheceu.

Queiroz (2000) enfatiza a necessidade do surgimento de serviços de apoio às famílias para o cuidado com os idosos. Segundo este autor, a família moderna passa, devido a questões econômicas e sociais, por uma transformação nos papéis, onde a mulher tem aumentado sua participação no mercado de trabalho, reduzindo ou eliminando a possibilidade de continuar sendo a tradicional cuidadora familiar. Aliado a isto, persiste o mito reforçado pela sociedade industrial de atribuir à família a obrigação do cuidado com suas avós, sogros, etc. Isto conduz à necessidade de arranjos na tentativa de evitar a institucionalização, onde pessoas de mais de uma geração se acomodam para tentar garantir melhor assistência aos idosos comprometidos.

As mudanças significativas da pirâmide populacional começam a acarretar uma série de previsíveis conseqüências sociais, culturais e epidemiológicas, para as quais ainda não estamos preparados para enfrentar. Tem-se desenvolvido, dentro deste contexto, uma rápida transição nos perfis de saúde em nosso país que se caracteriza, em primeiro lugar, pelo predomínio das enfermidades crônicas não-transmissíveis e, em segundo lugar, pela importância crescente de fatores de risco para a saúde e que requerem, complexamente, ações preventivas em diversos níveis. A medicina, associada à fisioterapia, muito tem a contribuir para uma melhor qualidade de vida dos idosos institucionalizados (CRUZ; ALHO, 2000).

Este estudo indicou que as concepções dos cuidadores em relação ao envelhecimento são constituídas, principalmente, por idéias de dependência dos idosos, sendo que o cuidado é descrito através da experiência de vida que cada cuidador ganha, e não como um trabalho profissional.

Monteiro e Faro (2006) encontraram resultados em sua pesquisa que apontam para o fato de que os cuidadores ainda são predominantemente informais, ou seja, indivíduos leigos na área da saúde, e que, portanto, necessitam de apoio de toda a equipe de saúde e informações sobre a realização de cuidados, bem como de orientações relativas à adaptação do ambiente ao idoso. 


\title{
PERCEPTION OF CAREGIVERS BEFORE THE DIFFICULTIES FOUND IN THE DAILY CARE OF INSTITUTIONALIZED DEPENDENT ELDERLY
}

\begin{abstract}
The health of aging people depends on many kinds of care, while old age makes people more vulnerable and claiming for aid. The purpose of this study was to verify the perception of caregivers concerning daily care problems found in dependent institutionalized elderly. This study was conducted in the Santo Antônio Asylum, in Cruz Alta (RS), a philanthropic institution, directed by the Conferência dos Vicentinos and internally managed by religious. The sample consisted of seven elderly's caregivers from the institution. The instruments used were: an identification form and a semi-structured interview with the caregivers, in attempt to verify what the caregiver think about the aging, and how they handle the care difficulties with the dependent institutionalized elderly. Information was recorded and then transcribed. For qualitative analysis of information the content's analysis technique was used. The answers indicate that caregivers have a vision about care for the elderly as something defined by experience, rather than a process of improvement and work. Regarding the conception of ageing reported by caregivers, they described ageing process mainly related with negative aspects of old age. The perceptions of caregivers describe the elderly as a dependent person, mainly when they are conducted to institutionalization. The reports suggested an old people similar to a concept of children. In conclusion, the study indicates that the conceptions of caregivers interviewed about the elderly and the ageing process are identified mainly by the dependency, and the care is defined by the experience of living of each caregiver.

Keywords: Ageing. Conception. Experience. Institutionalization. Dependence.
\end{abstract}

\section{REFERÊNCIAS}

BARDIN, Lawrence. Análise de Conteúdo. Lisboa: Persona, 1979.

BEAUVOIR, Simone de. A Velhice. 3. ed. Rio de Janeiro: Nova Fronteira, 1990.

Estud. interdiscip. envelhec., Porto Alegre, v. 13, n. 2, p. 259-274, 2008. 
BOFF, Leonardo. Saber Cuidar: ética do humano - compaixão pela Terra. Petrópolis: Vozes, 1999.

BORG, Christel; HALLBERG, Ingalill R.; BLOMQVIST, Kerstin. Life Satisfaction among Older People (65+) with Reduced Self-Care Capacity: the relationship to social, health and finantial aspects. Journal of Clinical Nursing, Oxford, v. 15, n. 5, p. 607-618, May 2006.

BORN, Tomiko; BOECHAT, Norberto Seródio. A Qualidade dos Cuidados ao Idoso Institucionalizado. In: FREITAS, Elizabete Viana de et al. (Org.). Tratado de Geriatria e Gerontologia. Rio de Janeiro: Guanabara Koogan, 2002. p. 768-777.

CALDAS, Célia Pereira. Envelhecimento com Dependência: responsabilidades e demandas da família. Cadernos de Saúde Pública, Rio de Janeiro, v. 19, n. 3, p. 733-781, jun. 2003.

CAMARANO, Ana Amélia. Os Novos Idosos Brasileiros: muito além dos 60? Rio de Janeiro: Ipea, 2004.

CARAVANA NACIONAL DE DIREITOS HUMANOS, 5., 2002, Brasília, DF. Uma amostra da realidade dos abrigos e asilos de idosos no Brasil: relatório. Brasília, DF: Comissão de Direitos Humanos, Câmara dos Deputados, 2002. Disponível em: <http:// www.dhnet.org.br/dados/caravanas/br/v_caravana.htm>. Acesso em: 25 nov. 2006.

CHAIMOWICZ, Flávio; GRECO, Dirceu B. Dinâmica da Institucionalização de Idosos em Belo Horizonte, Brasil. Revista de Saúde Pública, São Paulo, v. 33, n. 5, p. 454-460, out. 1999.

CREUTZBERG, Marion. Tratar Mais a Pessoa Idosa, Sobretudo a que Está Acamada: subsídios para o cuidado domiciliar. O Mundo da Saúde, São Paulo, v. 24, n. 4, jul./ago. 2000 .

CRUZ, Ivana Beatrice Mânica; ALHO, Clarice Sampaio. Envelhecimento Populacional: paradigma epidemiológico e de saúde do Brasil e do Rio Grande do Sul. In: JECKELNETO, Emílio Antônio; CRUZ, Ivana Beatrice Mânica (Org.). Aspectos Biológicos e Geriátricos do Envelhecimento. Porto Alegre: Edipucs, 2000. P. 175-191.

D’ALENCAR, Raimunda Silva. A Fabricação Social do Idoso e o Papel da Educação. Especiaria, Ilhéus, v. 2, p. 16-24, 1998.

DUARTE, Yeda Aparecida de Oliveira; DIOGO, Maria José. Atendimento Domiciliar: um enfoque gerontológico. São Paulo: Atheneu, 2000.

FARO JÚNIOR, Mário Paulo; LOURENÇO, Alexandre F.; BARROS NETO, Turibio Leite. Alterações Fisiológicas e Atividade Física na Terceira Idade: prescrição de exercícios. Ambito Medicina Desportiva, São Paulo, v. 6, n. 20, p. 5-10, 1996.

FERNANDEZ-BALLESTEROS, Rocio (Org.). Evaluación Conductual Hoy: un enfoque para el cambio en psicología clínica y la salud. Madrid: Pirámide, 1994. 
GRAEFF, Lucas. Instituições Totais e a Questão Asilar: uma abordagem compreensiva. Estudos Interdisciplinares sobre o Envelhecimento, Porto Alegre, v. 11, p. 9-27, 2007.

GRIMLEY EVANS, John. Geriatric Medicine: a brief history. BMJ, London, v. 315, n. 7115, p. 1075-1077, Oct. 1997.

KANE, Rosalie A.; KANE, Robert L. Long-Term Care: principles, programs and policies. New York: Springer, 1987.

KATZ, Paul R. Nursing Home Care In: JAHNIGEN, Dennis W.; SCHRIER, Robert. Geriatric Medicine. Cambridge: Blackwell Science, 1996. P. 175-186.

KHAW, Kay-Tee. Healthy aging. BMJ, v. 315, n. 7115, p. 1090-1096, Oct. 1997.

LEITE, Marines Tâmbara. A Velhice Pessoal no Imaginário de Estudantes de Enfermagem. Estudos Interdisciplinares sobre o Envelhecimento, Porto Alegre, v. 8, p. 115-124, 2005.

MENDONÇA, Jurilza Maria Barros. Instituição de Longa Permanência para Idosos e Políticas Públicas. Revista Kairós, São Paulo, v. 9, n. 2, p. 169-190, dez. 2006.

MONTEIRO, Carla Roberta; FARO, Ana Cristina Mancussi. O Cuidador do Idoso e sua Compreensão sobre a Prevenção e o Tratamento Cirúrgico das Fraturas do Fêmur. Estudos Interdisciplinares sobre o Envelhecimento, Porto Alegre, v. 10, p. 105-121, 2006.

NERI, Anita Liberalesso. Desenvolvimento e Envelhecimento: perspectivas biológicas, psicológicas e sociológicas. São Paulo: Papirus, 2001.

PRATO, Rosa et al. The Perception of Healthcare Quality of Elderly in the City of Bari, South Italy. BMC Health Services Research, London, v. 7, p. 174, Oct. 2007.

QUEIROZ, Zally Pinto Vasconcellos de. Cuidando do Idoso: uma abordagem social. O Mundo da Saúde, São Paulo, v. 24, n. 4, p. jul./ago. 2000.

REZENDE, Joffre M. Linguagem Médica: “institucionalização" do idoso. [S.1.]: J. M. Rezende, 2002. Disponível em: <http://usuarios.cultura.com.br/jmrezende/idoso.htm>. Acesso em: 13 ago. 2006.

RODRÍGUEZ, Josep A. Envejecimiento y Família. Madrid: Siglo XXI, 1994.

SILVA, Marco Antônio Pires Dias da. O Cuidar do Paciente Terminal: questões éticas. O Mundo da Saúde, São Paulo, v. 24, n. 4, p. 272-277, jul./ago. 2000 b.

SILVA, Iolete Ribeiro da; GUNTHER, Isolda de Araújo. Papéis Sociais e Envelhecimento em uma Perspectiva de Curso de Vida. Psicologia: Teoria e Pesquisa, Brasília, DF, v. 16, n. 1, p. 31-40, jan./abr. 2000a.

SINÉSIO, Neila Barbosa Osório; ANDRADE, Carmem Maria. Experiências Educativas numa Instituição Asilar. Praxisterapia, Cruz Alta, v. 2, n. 2, p. 21-29, dez. 2000. 
STREY, Marlene Neves et al. Velhice e Casamento: vivências e visões. Estudos Interdisciplinares sobre o Envelhecimento, Porto Alegre, v. 2, p. 23-34, 1999.

VAGETTI, Gislaine Cristina; WEINHEIMER, Marlei Salete; OLIVEIRA, Valdomiro de. Atendimento Integral à Saúde do Idoso Residente em Instituição de Longa Permanência: uma experiência interdisciplinar. Estudos Interdisciplinares sobre o Envelhecimento, Porto Alegre, v. 11, p. 53-66, 2007.

WALSH, Greg. Food, aged care and regional economics. Asia Pacific Journal of Clinical Nutrition, London, v. 13, p. S20, 2004. Supplement.

WORLD HEALTH ORGANIZATION. Active Ageing: a policy framework. Madrid, 2002. Available from: $<$ http://whqlibdoc.who.int/hq/2002/WHO_NMH_NPH_02.8.pdf $>$. Access at: 12 Sept. 2006.

Recebido em: 14-08-2008

$1^{a}$ revisão: $24-09-2008$

$2^{a}$ revisão: 29-01-2009

Aceite final: $30-01-2009$ 